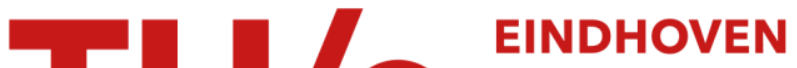 UNIVERSITY OF TECHNOLOGY
}

\section{Transverse diffusion in isotropic light slowing}

Citation for published version (APA):

Aardema, T. G., Knops, R. M. S., Nijsten, S. P. L., Leeuwen, van, K. A. H., Driessen, J. P. J., \& Beijerinck, H. C. W. (1996). Transverse diffusion in isotropic light slowing. Physical Review Letters, 76(5), 748-751. https://doi.org/10.1103/PhysRevLett.76.748

DOI:

10.1103/PhysRevLett.76.748

Document status and date:

Published: 01/01/1996

\section{Document Version:}

Publisher's PDF, also known as Version of Record (includes final page, issue and volume numbers)

\section{Please check the document version of this publication:}

- A submitted manuscript is the version of the article upon submission and before peer-review. There can be important differences between the submitted version and the official published version of record. People interested in the research are advised to contact the author for the final version of the publication, or visit the $\mathrm{DOI}$ to the publisher's website.

- The final author version and the galley proof are versions of the publication after peer review.

- The final published version features the final layout of the paper including the volume, issue and page numbers.

Link to publication

\section{General rights}

Copyright and moral rights for the publications made accessible in the public portal are retained by the authors and/or other copyright owners and it is a condition of accessing publications that users recognise and abide by the legal requirements associated with these rights.

- Users may download and print one copy of any publication from the public portal for the purpose of private study or research.

- You may not further distribute the material or use it for any profit-making activity or commercial gain

- You may freely distribute the URL identifying the publication in the public portal.

If the publication is distributed under the terms of Article 25fa of the Dutch Copyright Act, indicated by the "Taverne" license above, please follow below link for the End User Agreement:

www.tue.nl/taverne

Take down policy

If you believe that this document breaches copyright please contact us at:

openaccess@tue.nl

providing details and we will investigate your claim. 


\title{
Transverse Diffusion in Isotropic Light Slowing
}

\author{
T. G. Aardema, R. M. S. Knops, S. P. L. Nijsten, K. A. H. van Leeuwen, J. P. J. Driessen, and H. C. W. Beijerinck \\ Physics Department, Eindhoven University of Technology, P.O. Box 513, 5600 MB Eindhoven, The Netherlands
}

(Received 18 January 1995)

\begin{abstract}
We have demonstrated the slowing of a beam of cold $(180 \mathrm{~K})$ metastable neon atoms in a diffuse reflecting cylindrical cavity with monochromatic light. Using a well-collimated atomic beam entering the cavity, it is shown that stimulated emission has a major effect on the transverse velocity distribution. A large saturation parameter $s$ results in a loss mechanism for the center line beam intensity. A Monte Carlo simulation shows that the light intensity should be evenly distributed with moderate saturation conditions, to optimize the slowing effect while minimizing the transverse diffusion.
\end{abstract}

PACS numbers: $32.80 . P \mathrm{j}, 42.50 . \mathrm{Vk}$

Studying cold collisions requires dense samples of cold atoms. The method used so far has been to fill a trap with slow atoms that are subsequently cooled to the desired temperatures in the $\mathrm{mK}$ range. The collision processes are then probed by observing, e.g., the trap loss rate, or in more detail, the yield of fluorescence or electrons. Commonly used methods for slowing atoms down to velocities in the capture range of the trap $\left(\approx 10 \mathrm{~ms}^{-1}\right.$ for $\mathrm{Na}$ or metastable $\mathrm{Ne}$ ) are the Zeeman technique [1,2] and the chirped frequency technique [3,4]. Recently white light [5] and isotropic monochromatic light slowers [6,7] have been introduced as promising alternatives. Isotropic light slowers are interesting because of their simple implementation into an atomic beam line and the absence of both magnetic fields and an on-axis laser beam.

Monochromatic isotropic light slowing is based on geometric selection of resonant light through the Doppler effect. An atom with resonance frequency $\omega_{a}$ compensates for the laser detuning, $\delta=\omega_{l}-\omega_{a}$, by absorbing an incident photon from a cone with top angle $\theta=$ $\arccos (-\delta / k v)$ with respect to the atomic velocity $\vec{v}$ [see Fig. 1(a)], resulting in

$$
\omega_{a}=\omega_{l}+k v \cos \theta,
$$

with $k$ the magnitude of the wave vector. The corresponding velocity change, $v_{r}=\hbar k / m$, has components both parallel $(\cos \theta)$ and perpendicular $(\sin \theta)$ to the atomic velocity $\vec{v}$. The parallel component can be utilized for slowing the atomic beam. For red detuned light, $\delta<0$, we find $0<\theta<\pi / 2$, meaning that the photon recoil, $v_{r, \|}=\hbar k \cos \theta / m$, opposes the atomic motion. Subsequent spontaneous emission results in a zero photon recoil on average, and the atoms are slowed. As the atoms decelerate, the angle $\theta$ decreases until the minimum value of $\theta(=0)$ is obtained, corresponding to a final atomic velocity $v_{\delta}=-\delta / k$. The perpendicular component of the photon recoil, $v_{r, \perp}=\hbar k \sin \theta / m$, is zero on average. However, the statistical distribution of the azimuthal angle of the absorbed photons $\left[\varphi_{1}\right.$ in Fig. 1(a)] and the random nature of the spontaneous emission afterwards lead to an increase of the perpendicular velocity spread, $\left\langle\Delta v_{\perp}^{2}\right\rangle$, and contribute to the so-called transverse diffusion.
In contrast to Zeeman slowing, the absorptionstimulated emission cycle has to be taken into account in isotropic light slowing. Stimulated emission by a resonant photon from the cone cancels the slowing effect of the absorbed photon. In general the two photons have a different azimuthal angle, $\Delta \varphi=\varphi_{1}-\varphi_{2}$ (Fig. 1). This results in an extra transverse velocity change, $\left|\Delta v_{\perp}\right|=2 v_{r, \perp} \sin (\Delta \varphi / 2)$, and contributes to the transverse diffusion. The average contribution for one cycle is equal to

$$
\left\langle\Delta v_{\perp}^{2}\right\rangle_{\Delta \varphi}=2 v_{r, \perp}^{2}=2(\hbar k / m)^{2} \sin ^{2} \theta .
$$

We will refer to this extra transverse diffusion as stimulated diffusion. In this Letter we investigate the isotropic slowing of a beam of cold metastable neon atoms, including transverse diffusion.
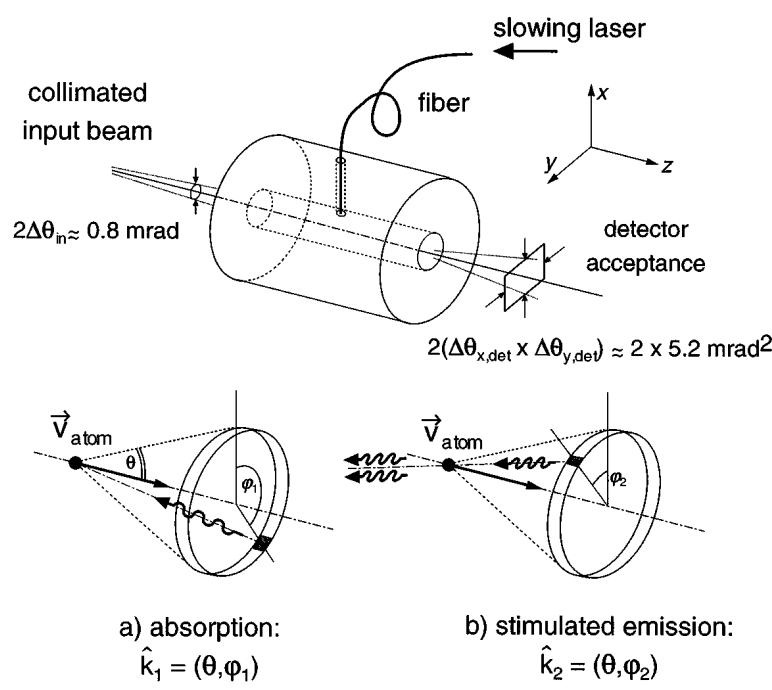

FIG. 1. The isotropic slower is a $31.5 \mathrm{~mm}$ long cylindrical Spectralon rod with an inner diameter of $1.5 \mathrm{~mm}$. Laser light is coupled into the cavity using a multimode fiber. Atoms entering the cavity are collimated to $\approx 0.8 \mathrm{mrad}$. An aperture in front of the detector determines the solid angle acceptance, $\approx 2 \times 5.2 \mathrm{mrad}^{2}$. Atoms absorb photons from a cone with top angle $\theta$ (a). The photon recoil parallel to the atomic velocity $\vec{v}$ is cancelled by stimulated emission (b). The azimuthal angles of the absorbed and the emitted photon, $\varphi_{1}$ and $\varphi_{2}$, result in a net velocity change of the atoms in the transverse direction. 
The isotropic slower is implemented experimentally using cylindrical sections of Spectralon [8] with a diffuse reflectivity $R \approx 0.99$. The outer diameter is $32 \mathrm{~mm}$; the length of a single section is $L=31.5 \mathrm{~mm}$. The bore has an inner diameter $d=1.5 \mathrm{~mm}$. Up to three sections can be lined up, thus enlarging the cavity length. Light is coupled into the cavity sections halfway using a multimode optical fiber (see Fig. 1). The intensity distribution $\Phi(z)$ of the laser light on the inside wall of the optical cavity is calculated in a Monte Carlo simulation [9], in which typically a thousand ray traces are calculated using a Lambertian reflection distribution [10]. We observe a nearly linear drop of $\Phi(z)$ from the center $(z=L / 2)$ to the outer ends $(z=0, L)$ by a factor of 40 . This decrease depends critically on the ratio $L / d$. This model can also reproduce the convoluted intensity distribution on the outside wall as measured by Batelaan et al. [7], who used a diameter of $6 \mathrm{~mm}$ instead of the reported $3 \mathrm{~mm}$ [11].

A CW ring dye-laser (Spectra Physics) is tuned near resonance to the neon two level transition at $\lambda=640.2 \mathrm{~nm}$. Saturated absorption at the atomic frequency $\omega_{a}$ is used to calibrate the laser frequency $\omega_{l}$. The laser frequency is locked to an electronically controlled external cavity, providing the desired frequency offset, $\delta=-2 \pi \times$ $750 \mathrm{MHz}\left(v_{\delta}=480 \mathrm{~ms}^{-1}\right)$. No active long-term drift corrections for the laser frequency are made. The maximum frequency drift is estimated at $\approx 10 \mathrm{MHz} / \mathrm{h}$, which is acceptable with typical measuring times of $10 \mathrm{~min}$ for a velocity distribution.

A beam of cold metastable $\mathrm{Ne}^{*}$ atoms is passed through the cavity to determine the influence of the isotropic light field on both the axial and perpendicular velocity distribution. The $\mathrm{Ne}^{*}$ atoms are produced in a discharge excited supersonic expansion source, cooled with liquid nitrogen to an effective temperature of $T \approx$ $180 \mathrm{~K}$ [12], corresponding with a mean velocity of $v \approx$ $560 \mathrm{~ms}^{-1}$ and a velocity spread of $\Delta v \approx 130 \mathrm{~ms}^{-1}$. The final velocity distribution $I(v) \mathrm{d} v$ is determined using the time-of-flight (TOF) method with a mechanical chopper and a metastable atom detector, which are located 790 and $1760 \mathrm{~mm}$ downstream of the isotropic slower, respectively. The velocity resolution, $\Delta v_{\mathrm{TOF}}=20 \mathrm{~ms}^{-1}$ at $v \approx 500 \mathrm{~ms}^{-1}$, is determined by the width of the slit in the mechanical chopper [13]. To measure the effect of transverse diffusion on the perpendicular velocity distribution, we collimate the atomic beam to $2 \Delta \theta_{\text {in }} \approx$ $0.8 \mathrm{mrad}$, i.e., a perpendicular velocity spread $\Delta \boldsymbol{v}_{\perp} \approx$ $0.2 \mathrm{~ms}^{-1}$. The rectangular solid acceptance angle of the metastable atom detector is $2\left(\Delta_{\operatorname{det}, x} \times \Delta_{\operatorname{det}, y}\right) \approx 2 \times$ $5.2 \mathrm{mrad}^{2}$, corresponding to a velocity acceptance $\Delta v_{\perp} \approx$ $0.5 \mathrm{~ms}^{-1}$ in the $x$ direction. Note that $v_{r}=0.03 \mathrm{~m} / \mathrm{s}$ for neon. Transverse diffusion can thus result in a loss of detected metastable atoms.

The measured velocity distributions for three values $3 \times$ $2.5,3 \times 10$, and $3 \times 40 \mathrm{~mW}$ of input power are shown in Fig. 2. Three sections are aligned, each equipped with a separate optical fiber. As a reference the initial velocity distribution is given, as measured with the laser switched "off." Note that $25 \%$ of the detected metastable atoms is not affected by the light: it is the wrong isotope, $10 \%$ ${ }^{22} \mathrm{Ne}^{*}$, or a different metastable level, $15 \%{ }^{20} \mathrm{Ne}^{*}\left({ }^{3} P_{0}\right)$. The effect of slowing is obvious. Atoms with a higher initial velocity are slowed towards the desired final velocity $v_{\delta}=480 \mathrm{~ms}^{-1}$ resulting in a sharp peak in the laser "on" distributions. The width of the peaks is $\Delta v \approx 20 \mathrm{~ms}^{-1}$, in agreement with $\Delta v_{\text {TOF. }}$. Because of the drift of $\omega_{l}$ in time between the measurements, the frequency shift $\delta$ is slightly different for the three power settings. The height of the slowed peak hardly increases with increasing laser power.

The most remarkable feature is the discrepancy in the area above and below the initial velocity distribution when comparing the on and off situations: Some of the atoms are obviously lost when they are decelerated. For higher input powers the difference becomes even more pronounced. To investigate this loss of atoms we have performed preliminary measurements with a 1D position sensitive detector, replacing the current detector of the atomic beam. We observe that the beam profile with the slower switched on has a different character: broad "wings" are visible, together with a loss of atoms from the narrow central peak of the original atomic beam. This suggests that transverse diffusion beyond the $2 \mathrm{mrad}$ acceptance angle in the $x$ direction of the atomic beam detector is the main cause of the loss of atoms visible in Fig. 2.

To investigate the above effect we simulate the atomic trajectories in the slower with a Monte Carlo calculation. All atoms start on axis and thus see a cylindrical slice of the cavity wall with resonant light [Fig. 1(a)]. The effective width of the slice is determined by the Lorentzian profile of the transition. The energy density distribution $U(\omega, z) d \omega$

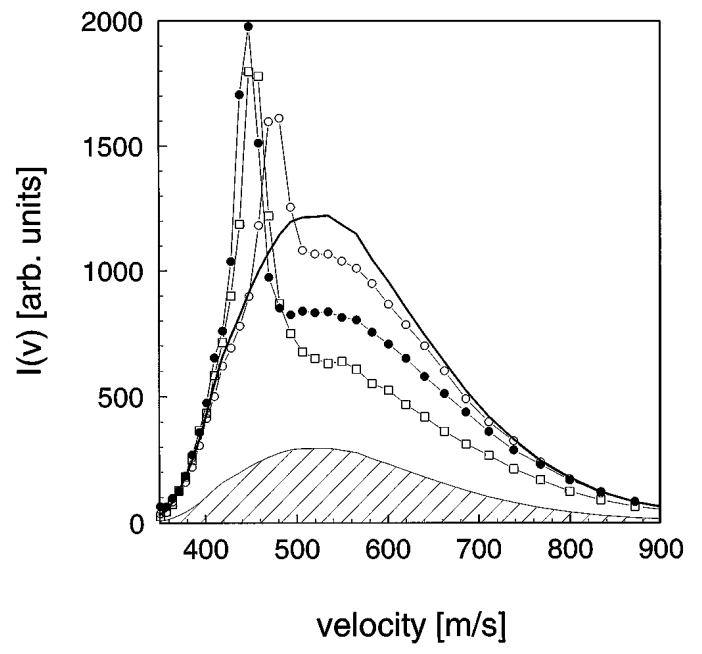

FIG. 2. Experimental results for velocity distributions with "laser on" and "off," obtained with three Spectralon sections. The detuning is $\delta=-2 \pi \times 750 \mathrm{MHz}\left(v_{\delta}=480 \mathrm{~ms}^{-1}\right)$. The laser powers used are $3 \times 2.5 \mathrm{~mW}(\circ), 3 \times 10 \mathrm{~mW}(\bullet)$, and $3 \times 40 \mathrm{~mW}(\square)$. The solid line represents the initial distribution (laser off). The hatched area represents the $25 \%$ fraction of the metastable atoms which are unaffected by the laser light, i.e., ${ }^{22} \mathrm{Ne}^{*}$ and $\mathrm{Ne}^{*}\left({ }^{3} P_{0}\right)$. 
experienced by the moving atom can be derived from the calculated light intensity $\Phi(z)$ on the cavity wall using the Doppler effect. Therefore the rates of absorption and stimulated and spontaneous emissions can be calculated using the Einstein coefficients and the energy density in the cavity [14]. Along the trajectory, the time evolution of the internal state of the atom is calculated according to the stochastic nature of absorption and emission, taking into account the corresponding rates. This also holds for the choice whether stimulated or spontaneous emission will occur. Note that the rates which determine these events are $z$ dependent. The distribution function of final velocities gives both the deceleration as well as the transverse diffusion of the atoms. By choosing $\varphi_{1}=\varphi_{2}$ (Fig. 1) we can switch off the effect of an absorptionstimulated emission cycle on the atomic motion, without modifying the time evolution of the internal state of the atom.

In the Monte Carlo calculation we have taken input powers $3 \times 2.5$ and $3 \times 40 \mathrm{~mW}$ for the three cavities. The laser detuning is $\delta=-2 \pi \times 750 \mathrm{MHz}$. The results of the calculation are shown as a function of the initial atomic velocity $v$ in Figs. 3(a) (the average final velocity) and 3(b) (the rms value of the deflection angle).

The average final velocities in Fig. 3(a) clearly demonstrate a broad initial velocity range being transformed to a narrow range of final velocities, $\approx v_{\delta}$. The width of this broad range, $v-v_{\delta}$, is called the capture range and is found to be $\approx 35$ and $\approx 96 \mathrm{~ms}^{-1}$ for the $3 \times 2.5$ and $3 \times 40 \mathrm{~mW}$ cases, respectively. The capture range depends on both the interaction time in the cavity, $\approx L / v_{\delta}$, and the deceleration, $\approx v_{r, \|} \Gamma / 2$, with $\Gamma$ the spontaneous emission rate. An upper estimate for the capture range is given by

$$
\left(v-v_{\delta}\right)_{\max }=\left(L \Gamma / 2 v_{\delta}\right) v_{r},
$$

which equals $152 \mathrm{~ms}^{-1}$ for three sections with $L=$ $94.5 \mathrm{~mm}$. Because of our method of switching off the effect of absorption-stimulated emission cycles, as discussed previously, Fig. 3(a) is unaffected by this action.

As a result of the transverse diffusion the atoms are deflected over an angle $\theta=v_{\perp} / v_{f}$. The average deflection angle as a function of the initial velocity is shown in Fig. 3(b). The rectangular aperture, $1.5 \times$ $4 \mathrm{~mm}$, positioned $765 \mathrm{~mm}$ downstream of the isotropic slower results in a loss factor. This loss factor is calculated for each deflection angle and is shown on the right-hand $y$ axis. Comparing Figs. 3(a) and 3(b) we see that the position of maximum deflection corresponds to the fastest atoms just within the capture range. The resonance condition for these atoms results in a rather large initial angle $\theta$, resulting in a fairly large contribution from stimulated diffusion $\left(\propto \sin ^{2} \theta\right)$. For initial velocities above the capture range the angle $\theta$ becomes so large that the average photon recoil, $\hbar k \cos \theta / m$, is insufficient to slow the atom to the desired final velocity $v_{\delta}$.

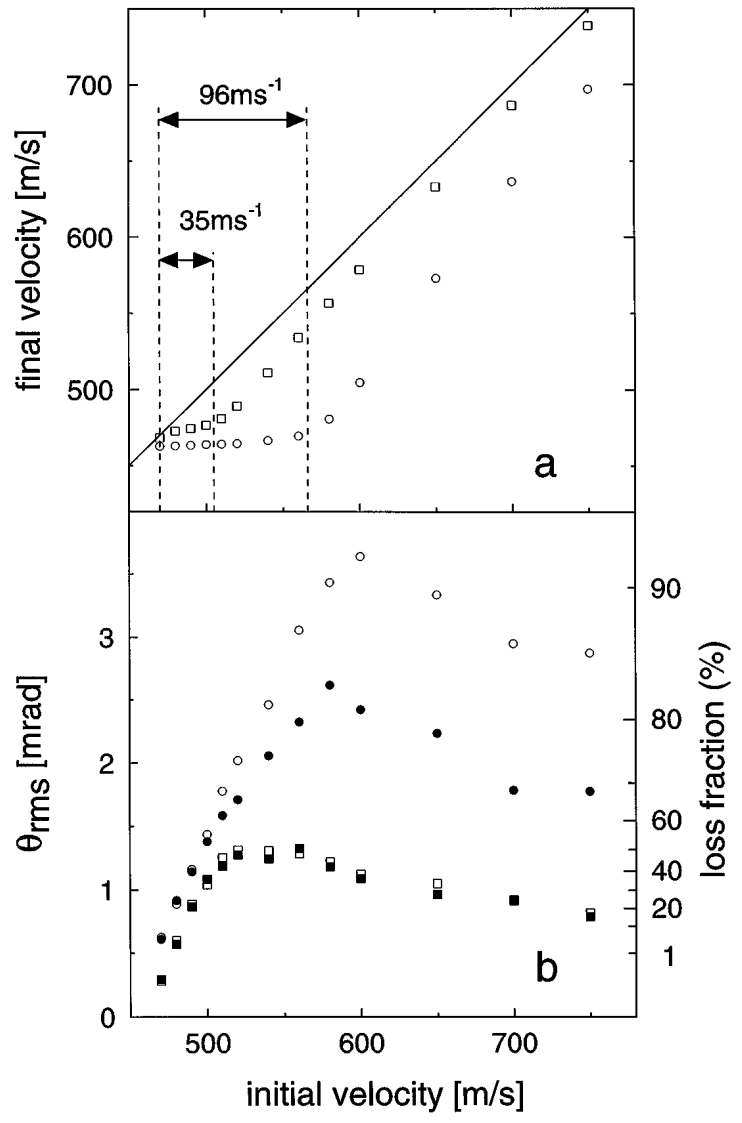

FIG. 3. Monte Carlo calculation of (a) the average final velocity and (b) the rms value of the deflection angle versus the initial velocity. Shown are two input power calculations, $3 \times 2.5 \mathrm{~mW}$ (circles) and $3 \times 40 \mathrm{~mW}$ (squares). The capture range, which gives a $10 \mathrm{~ms}^{-1}$ wide $v_{\mathrm{f}}$ range, has been indicated for both cases. The significant role of stimulated diffusion is demonstrated in (b). The deflection angle is calculated both with (empty markers) and without (filled markers) including the stimulated emission process. The right-hand axis in (b) gives the loss fraction of the atoms due to the final aperture (Fig. 1).

The importance of stimulated diffusion is also obvious from Fig. 3(b), where the results of the Monte Carlo calculation with and without stimulated emission are displayed. In the $3 \times 2.5 \mathrm{~mW}$ case we observe a negligible difference, indicating that spontaneous emission is the major driving force of the transverse diffusion. However, for the $3 \times 40 \mathrm{~mW}$ case there is an extra contribution of 1.0 mrad due to stimulated diffusion, which is also prominent within the capture range. Higher laser power has the negative side effect of a large increase in transverse diffusion.

If the goal is to enlarge the capture range and simultaneously reduce transverse diffusion, one should maximize the number of absorption-spontaneous emission cycles and minimize the number of absorption-stimulated emission cycles. The number of cycles scale as $\sim s /(s+$ 1) for spontaneous emission and as $\sim s^{2} /(s+1)$ for stimulated emission, with $s$ the on-resonance saturation parameter as defined in a two level system. From 
the calculated intensity distribution $\Phi(z)$ the value of $s(z)$ is found to vary between $0.01<s<0.9$ and $0.2<s<15.3$ for the $3 \times 2.5 \mathrm{~mW}$ and $3 \times 40 \mathrm{~mW}$ cases, respectively. It is evident that the $3 \times 40 \mathrm{~mW}$ situation is dominated by the absorption-stimulated emission cycles.

An alternative manner to enlarge the capture range is elongating the cavity by using multiple cavity sections. The laser power per section can now be chosen such that stimulated diffusion has a negligible effect. For large velocities in the capture range the resonance condition of Eq. (1) results in large angles $\theta$ : To avoid the average photon recoil, $\hbar k \cos \theta / m$, from becoming too small, we might use different laser detunings in the various cavities thus keeping $\cos \theta$ as large as possible throughout the whole cavity as has been demonstrated by Ketterle et al. [6].

To compare our model with experimental results we need to construct a velocity distribution from our calculated results of Figs. 3(a) and 3(b). The initial parallel velocity distribution is transformed to a slowed spectrum using the calculated deceleration. Both the time of flight velocity resolution and the finite solid angle acceptance of the detector have been taken into account. This results in a velocity distribution with a narrow slowed peak at a slightly shifted velocity $v_{f}=v_{\delta}-2 \Gamma / k$; the extra deceleration results from the Lorentz linewidth, or so-called off-resonance slowing [6].

For the $3 \times 10 \mathrm{~mW}$ case, the calculated distribution is compared with the experimental results in Fig. 4, giving nice agreement. Because $\Phi(z)$ scales with $(1-\mathcal{R})^{-1}$, this calculation is extremely sensitive for the reflectivity $\mathcal{R}$ of the cylindrical Spectralon cavity: An increase in

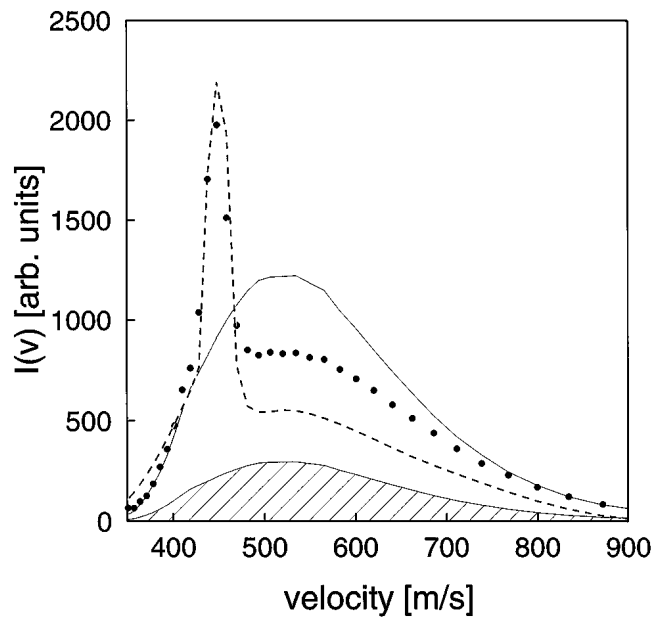

FIG. 4 Calculated distribution with a detuning of $\delta=-2 \pi \times 720 \mathrm{MHz}$, for three Spectralon sections. The thin solid line represents the measured initial distribution with laser off. The dashed line is the calculation for the $3 \times 10 \mathrm{~mW}$ case; the experimental data with laser on are indicated by the dots. The hatched area represents the $25 \%$ fraction of the metastable atoms which are unaffected by the laser light. reflectivity by $\approx 0.8 \%$ results in a loss factor $>0.9$ for velocities $v>500 \mathrm{~m} / \mathrm{s}[7,15]$.

This work demonstrates the isotropic slowing effect for $\mathrm{Ne}^{*}$ in a small cavity with low laser powers. Although papers [6,7] stated that this could be a promising technique for beam slowing, the effect of transverse diffusion caused by stimulated emission can destroy the beam quality dramatically. In the experiment of Batelaan et al. [7] the perpendicular velocity resolution $\left(v_{\perp} \approx \Delta 2 \mathrm{~ms}^{-1}\right)$ is too large for this effect to be observable. In the experiment of Ketterle et al. [6] the perpendicular velocity is not resolved at all. Through a careful design of the cavity [15], the effect of transverse diffusion can be minimized. In the setup described above the saturation parameter still varies over fairly large range $\left(s_{\max } / s_{\min } \approx 20\right)$. The laser light can be more evenly distributed along the $z$ axis, e.g., by illuminating the cylindrical cavity at more positions, spaced at closer intervals. Alternative ways of minimizing the transverse diffusion can be realized by illuminating the cylindrical cavity along the whole axis [11], or using a spherical cavity. In these cases, the intensity distribution in the cavity has no longer a peaked structure.

We wish to acknowledge helpful discussions with Dr. H. Batelaan. This work is financially supported by the Dutch Foundation for Fundamental Research on Matter (FOM). The research of J.D. has been made possible by a fellowship of the Royal Netherlands Academy of Arts and Science.

[1] W. D. Phillips, J. V. Prodan, and H. J. Metcalf, J. Opt. Soc. Am. B. 2, 1751 (1985).

[2] T. E. Barrett, S. W. Dapore-Schwartz, M. D. Ray, and G. P. Lafyatis, Phys. Rev. Lett. 67, 3483 (1991).

[3] V. I. Balykin, V.S. Letokhov, and V. I. Mishin, Zh. Eksp. Teor. Fiz. 78, 1376 (1980) [Sov. Phys. JETP 51, 692 (1980)].

[4] W. Ertmer, R. Blatt, J. L. Hall, and M. Zhu, Phys. Rev. Lett. 54, 996 (1985).

[5] M. Zhu, C. W. Oates, and J. L. Hall, Phys. Rev. Lett. 67, 46 (1991).

[6] W. Ketterle, A. Martin, M. A. Joffe, and E. Pritchard, Phys. Rev. Lett. 69, 2483 (1992).

[7] H. Batelaan, S. Padua, D. H. Yang, C. Xie, R. Gupta, and H. Metcalf, Phys. Rev. A 49, 2780 (1994).

[8] Labsphere Inc., P.O. Box 70, North Sutton, NH 03260.

[9] M. P. J. M. Stevens, H. C. W. Beijerinck, and N. F. Verster, Physica (Amsterdam) 83C, 209 (1976).

[10] M. Born and E. Wolf, Principals of Optics (Pergamon, New York, 1975), p. 182.

[11] H. Batelaan (private communication).

[12] M.D. Hoogerland, Ph.D. thesis, Eindhoven University of Technology, 1993.

[13] E. R. T. Kerstel, C.P. J.W. van Kruijsdijk, J.C. Vlugter, and H. C. W. Beijerinck, Chem. Phys. 121, 211 (1988).

[14] R. C. Hilborn, Am. J. Phys. 50, 982 (1982).

[15] T. G. Aardema, R. M. S. Knops, and H. C. W. Beijerinck (to be published). 